\title{
Returning to non-entailed presuppositions again ${ }^{1}$ \\ Jérémy ZEHR — University of Pennsylvania \\ Florian SCHWARZ — University of Pennsylvania
}

\begin{abstract}
Recent work by Sudo (2012) and Klinedinst (2016) proposes a new perspective on differences between classes of presupposition triggers, with an empirical split roughly mirroring Abusch's (2002) hard vs. soft distinction and related notions. These two authors propose that triggers differ in whether or not their presuppositional content simultaneously affects the calculation of the presuppositions and of the entailments of the sentences in which they appear. Drawing on a proposal by Glanzberg (2005) we formulate the Removability/Independence Hypothesis: triggers that do not affect entailments are triggers that can be left out of sentences without affecting interpretability. We experimentally test the hypothesis by embedding return, (go) again and (go) back in non-monotonic environments, which Sudo argues to elicit differences in presuppositions and entailments. Our results provide clear evidence against the RI hypothesis: whereas only the trigger return is crucial for the sake of interpretability, all three triggers produced similar results. At the same time, data for the triggers stop and also, included as controls, lend further support in favor of Sudo's entailment-contrast proposal.
\end{abstract}

Keywords: presuppositions, entailment, hard/soft distinction.

\section{Introduction}

As evident from (1), start, stop and too all trigger a presupposition: while a speaker can ask (1a) and remain agnostic as to whether Mei fought criminals before (and after) she graduated, they make a commitment about her pre-graduation habits when asking (1b), (1c) or (1d). Indeed, despite the interrogative aspect of these sentences, a speaker asking (1b) takes for granted that Mei did not fight criminals before graduating and, likewise, a speaker asking (1c) or (1d) takes for granted that she did fight criminals before she graduated. The presupposition is said to project: it survives embedding under a question operator. ${ }^{2}$

(1) a. Did Mei fight criminals after she graduated?

b. Did Mei start fighting criminals after she graduated?

c. Did Mei stop fighting criminals after she graduated?

d. Did Mei fight criminals after she graduated too?

Since Abusch (2002), it has been standard to assume a division between so-called hard and soft presuppositions. Abusch proposes that there is a categorical difference between presuppositions like those of start and stop on the one hand, and presuppositions like those of too on the other hand. She remarks that even though start and stop associate with contradictory presuppositions, a speaker can utter (2a) without suffering contradiction, with the resulting interpretation

\footnotetext{
${ }^{1}$ We gratefully acknowledge support from NSF-grant BCS-1349009 to Florian Schwarz. We thank the audience at the workshop Theoretical and Experimental Approaches to Presuppositions in Genoa, the audience at Sinn und Bedeutung 22, and the members at the lab meeting at UPenn for their comments.

${ }^{2}$ The phrase after she graduated arguably also introduces the presupposition that Mei graduated. We will ignore this in the rest of this paper.
} 
in (2b). This stands in contrast with (3a), where the presuppositions that too triggers (that she fought/supported criminals after she graduated) from each disjunct also stand in a relation of contradiction, thereby making (3a) a contradictory utterance. Abusch's conclusion is that start and stop are soft triggers whose presuppositions can easily be suspended for the sake of discourse felicity (as in (2)) whereas too is a hard trigger whose presuppositions project even when leading to discourse contradiction as in (3).

a. Mei either started fighting criminals after she graduated, or stopped fighting criminals after she graduated.

b. Mei either [didn't fight criminals before she graduated and] started afterwards, or [fought criminals before she graduated and] stopped afterwards. $=\quad(2 \mathrm{a})$

(3) a. ? Mei either supported criminals after she graduated too, or fought criminals after she graduated too.

b. Mei either [supported criminals before she graduated and] did so afterwards too, or [fought criminals before she graduated and] did so afterwards too. $\quad \neq$

Granted that presuppositions come either as hard or soft, the problem is twofold: i) what are the characteristics of presuppositions that make them easily suspendable (soft) or more persistent (hard) and ii) what systematic principles, if any, govern the mapping of triggers onto being either hard or soft? Abusch only offers an answer to the first question. ${ }^{3}$ She proposes that soft presuppositions are derived through pragmatic means, whereas hard presuppositions are encoded into the semantics of their lexical triggers. The assumption is that contextual factors can block the pragmatic derivation of a presupposition but cannot obviate the constraints that lexical items impose on the compositional computation of a sentence's semantic value.

While Abusch's proposal constitutes an explanatory account of why soft and hard presuppositions differ in terms of suspendability, it leaves the second aspect of the problem unresolved, namely what properties of expressions associate them with soft rather than hard (e.g., stop), or hard rather than soft presuppositions (e.g., too) ${ }^{4}$ In this paper, we consider a proposal by Klinedinst (2016: see also Sudo 2012) that develops an answer to the first question independently from Abusch's analysis, and a proposal by Glanzberg (2005: see also Zeevat 1992) that offers a possible answer to the second question. We consider the possibility of linking the two proposals by formulating what we call the Removability/Independence Hypothesis and proceed to experimentally test its predictions. While our results support the existence of a contrast opposing the trigger stop to the triggers also and again along a Sudo-Klinedinst split line, they reveal that the trigger return patterns with also and again rather than with stop, contra the predictions of our Removability/Independence Hypothesis. As a conclusion, we give a critical review of some of the current theories of presuppositions in light of our results.

\footnotetext{
${ }^{3}$ Though see her footnote 5 for hints at possible answers to the second question.

${ }^{4}$ From this point on we extend Abusch's terminology of soft vs. hard presuppositions in a way that respectively associates them with soft vs. hard triggers.
} 


\section{Entailing vs. Non-Entailing Presupposition Triggers}

\subsection{Theoretical Background}

In this paper, we will adopt Heim and Kratzer's (1998) notation of semantic values, which distinguishes domain conditions (in boldface, our emphases) from truth conditions (underlined, our emphases). (4) illustrates this notation: (4b) is a formal representation of the semantic value of (4a). The presupposition that Mei used to fight criminals before graduation is represented by the boldface domain condition. The underlined truth conditions represent the proposition about Mei fighting criminals after graduation. Operators like interrogation or disjunction target truth conditions but leave domain conditions unaffected, resulting in presupposition projection.

(4) a. Mei fought criminals after she graduated too.

b. $\quad[[(4 \mathrm{a})]]=\lambda w: \exists \mathbf{t}<\mathbf{t}_{\text {grad }}$ fight-criminals(m,t, w). fight-criminals $\left(m, t_{\text {grad }}, w\right)$.

In (2), the second disjunct containing the trigger stop, repeated in (5a), appears to lack a presupposition. We represent its apparent semantic contribution to (2a) in (5b): what appears as a domain condition in (4b) appears as part of the truth-conditions in (5b).
a. Mei stopped fighting criminals after she graduated.
b. $\quad \lambda w . \underline{\exists t<t_{\text {grad }} \text { fight-criminals }(m, t, w)} \wedge \quad \neg$ fight-criminals $\left(m, t_{\text {grad }}, w\right)$.

Were we unaware of the presuppositional properties of stop, a natural conclusion from the observation of (5a) receiving the interpretation in $(5 \mathrm{~b})$ would be to say that stop conveys only the truth conditions in (6).

$$
\lambda P . \lambda x . \lambda w . \exists t<t_{0} P(x, t, w) \wedge \neg P\left(x, t_{0}, w\right) .
$$

However, we have already established with (1c) that stop presupposes that its complement predicate was true of its subject at a previous time. A possible refinement would be to say that (7) is a more accurate representation of the lexical entry of stop, and posit an operation that can convert domain conditions into truth-conditions, which would derive (6) from (7). Speakers using (5a) in the disjunction (2a) could then apply that operation, and standard rules of composition would associate the stop disjunct with the semantic value in (5b).

$$
\lambda P . \lambda x . \lambda w: \exists \mathbf{t}<\mathbf{t}_{\mathbf{0}} \mathbf{P}(\mathbf{x}, \mathbf{t}, \mathbf{w}) . \neg P\left(x, t_{0}, w\right) .
$$

What we just described has been a standard approach since Heim (1983), who introduces this notion of conversion as local accommodation. It facilitates starting from a lexical representation of stop as in (7) so as to account for presuppositional uses as in (1c) while also accounting for non-presuppositional uses as in (2a) at the same time.

One pitfall of this approach in light of the contrast elicited by Abusch is that, as things stand, it would predict non-presuppositional uses of too to the same extent as there are nonpresuppositional uses of stop. If local accommodation can convert any domain condition into 
truth condition, then (4a) should also associate with an alternative version of (4b) where the boldface domain conditions appear as part of the underlined truth conditions, in a way parallel to (5b). A direct, unwelcome prediction is that the too disjunction (3a) should receive the non-presuppositional interpretation in (3b) in the same way that the start/stop disjunction (2a) receives the non-presuppositional interpretation in (2b). Since this is not the case, the only solution to account for the contrast is to stipulate that too resists local accommodation in a way that stop (and start) do not.

Klinedinst (2016) offers an alternative explanation. Rather than revising (6) as a semantic value for stop in favor of (7), Klinedinst proposes to enrich it with a domain condition as in (8).

$$
[[\text { stop }]]=\lambda P . \lambda x . \lambda w: \exists \mathbf{t}<\mathbf{t}_{\mathbf{0}} \mathbf{P}(\mathbf{x}, \mathbf{t}, \mathbf{w}) . \exists t<t_{0} P(x, t, w) \wedge \neg P\left(x, t_{0}, w\right) .
$$

Such a lexical entry does not straightforwardly account for apparent non-presuppositional uses of stop, and Klinedinst therefore also needs to posit an operation to prevent domain conditions from projecting and giving rise to a presupposition in the case of the stop-start disjunction (2a). One could make use of the operation of conversion that we described earlier, but note that that operation of conversion can be described as a two-step process: first make a copy of the domain conditions into the truth conditions, second delete the domain conditions. Only the second step is required when starting from the lexical entry in (8). (9) illustrates the one-step process.

$$
\begin{array}{lll}
\text { a. } & \lambda P . \lambda x . \lambda w: \exists \mathbf{t}<\mathbf{t}_{\mathbf{0}} \mathbf{P}(\mathbf{x}, \mathbf{t}, \mathbf{w}) . \exists t<t_{0} P(x, t, w) \wedge \neg P\left(x, t_{0}, w\right) . & \\
\text { b. } & \lambda P . \lambda x . \lambda w: \exists \mathbf{t}<\mathbf{t}_{\mathbf{0}} \mathbf{P}(\mathbf{x}, \mathbf{t}, \mathbf{w}) . \exists t<t_{0} P(x, t, w) \wedge \neg P\left(x, t_{0}, w\right) . & \text { DELETION } \\
\text { c. } & \lambda P . \lambda x . \lambda w . \exists t<t_{0} P(x, t, w) \wedge \neg P\left(x, t_{0}, w\right) .
\end{array}
$$

In our view, the main advantage of Klinedinst's approach over the standard approach does not come from reducing the complexity of the posited operation. After all, the price to pay for a simpler operation is to posit a richer, somewhat redundant lexical entry for stop: the same proposition appears both as a domain condition and as a truth condition. The very welcome consequence of Klinedinst's position becomes evident when one considers non-redundant semantic values, that is, semantic values where domain condition propositions do not appear as part of the truth conditions. Let us compare the alternative semantic values to the one we gave for the too disjunct in (4b) after conversion (10) versus after deletion (11).

(10) Mei fought criminals after she graduated too.

$$
\begin{array}{lr}
\text { a. } & \lambda w: \exists \mathbf{t}<\mathbf{t}_{\text {graduate }} \text { fight-criminals }(\mathbf{m}, \mathbf{t}, \mathbf{w}) . \\
\text { b. } & \lambda w: \exists \mathbf{t}<\mathbf{t}_{\text {graduate }} \text { fight-criminats }(\mathbf{m}, \mathbf{t}, \mathbf{w}) . \\
& {\left[\exists t<t_{\text {graduate }} \text { fight-criminals }(m, t, w)\right]_{\text {copy }} \wedge \text { fight-criminals }\left(m, t_{\text {graduate }}, w\right) .} \\
\text { c. } & \lambda w . \quad \exists t<t_{\text {graduate }} \text { fight-criminals }(m, t, w) \wedge \text { fight-criminals }\left(m, t_{\text {graduate }}, w\right) .
\end{array}
$$

(11) Mei fought criminals after she graduated too.
a. $\quad \lambda w: \exists \mathbf{t}<\mathbf{t}_{\text {graduate }}$ fight-criminals $(\mathbf{m}, \mathbf{t}, \mathbf{w})$. fight-criminals $\left(m, t_{\text {graduate }}, w\right)$.
b. $\quad \lambda w: \exists \mathbf{t}<\mathbf{t}_{\text {graduate }}$ fight-criminals $(\mathbf{m}, \mathbf{t}, \mathbf{w}) . \overline{\text { fight-criminals }\left(m, t_{\text {graduate }}, w\right)}$. DEL.
c. $\lambda w$. fight-criminals $\left(m, t_{\text {graduate }}, w\right)$. 
It should not come as a surprise that the truth conditions expressed in (10c) correspond exactly to the second disjunct of the unavailable interpretation (3b): the standard approach has to prevent conversion from happening with too in order to account for the unavailability of (3b). On the other hand, there is no way to arrive at that interpretation from (11c), since the proposition expressed in the domain condition has been lost in the process of deletion. This result makes Klinedinst's approach a better candidate to explain the contrast between stop and too than the standard approach. Klinedinst's view directly predicts a split along the lines of the hard vs. soft distinction by exhausting the answers to a question that arises when considering Heim and Kratzer's notation: do the propositions in the domain conditions also appear in the truth conditions? The answer is: when they do, the result is a soft trigger like stop and when they do not, the result is a hard trigger like too.

In this paper, we will refer to triggers like stop whose truth conditions entail their domain conditions as entailing triggers, and to triggers like too as non-entailing triggers. ${ }^{5}$ Klinedinst (2016) is not the first author to propose that one should distinguish between entailing and nonentailing triggers. This idea has received direct or indirect support both from the theoretical and the experimental literature.

\subsection{Theoretical support}

Two studies on the licensing of Negative Polarity Items (NPIs) have made use of the entailing vs. non-entailing distinction. Chierchia (2015: pp. 8-9) cites Gajewski (2011) as offering an analysis of the NPI-licensing contrast between plural and singular the illustrated in (12) along the lines of the entailing vs. non-entailing distinction. Gajewski models the plural and singular determiners as sharing an existential and a maximality presupposition, but models only singular the as also entailing the existence presuppositions. Gajewski then shows how the DownwardEntailing (DE) context characterizing the entailments of plural the gets neutralized by adding the presupposed content to the entailments of singular the. Since NPIs are assumed to be licensed only in Downward-Entailing (DE) contexts, only plural the licenses NPIs.

a. Plural: The clients that had any complaint were refunded.

b. Singular: * The client that had any complaint was refunded.

Drawing on Gajewski's proposal, Chierchia (2015) argues that such an entailing-vs-nonentailing approach can account for a contrast between English and Italian factives: while in English, certain emotive factives, but not cognitive factives, license NPIs (13), in Italian, no factive ever licenses NPIs, no matter whether emotive or cognitive. Chierchia assumes a crosslinguistically uniform semantics for factive verbs where only emotive factives introduce a DE context, thus accounting for their licensing of NPIs in English. He locates the contrast between English and Italian in their different complementizer systems (e.g., English that vs. Italian che). In the same way that the DE context found in the (non-entailing) plural determiner is neutralized by the (entailing) singular determiner in Gajewski (2011), Chierchia proposes that English

\footnotetext{
${ }^{5}$ Note that independently of Klinedinst's analysis, one can still conceive of an entailing vs. non-entailing contrast as technically distinct from the soft vs. hard distinction.
} 
and Italian complementizers both trigger the presupposition that the complement is true, but Italian complementizers additionally entail the truth of the complement. As a result, Chierchia shows, the DE context introduced by emotive factives get neutralized in Italian, but is left unaffected in English.

a. Emotive: She was surprised that there was any food left

b. Cognitive: * She was aware that there was any food left

Sudo (2012) already hints at Klinedinst's later analysis of the hard vs. soft contrast in terms of an entailing vs. non-entailing split, but his main interest lies in the consequences of such a theoretical split on truth value judgments. He makes a crucial contribution to this question by identifying a linguistic environment where the contrast becomes evident. Sudo observes that embedding stop or again under non-monotone quantifiers such as Exactly $N$ predicts different outcomes in otherwise equivalent contexts. Imagine two Linux computers and two Windows computers: the two Linux computers always crashed at launch last week, but the two Windows computers never crashed. This week, it was one of the two Linux computers and one of the two Windows computers that always crashed at launch. Sudo claims that the sentence (14) is a better description of the situation than the sentence (15), which he attributes to them having the semantic effect paraphrased in (b) for both examples.

(14) Exactly one computer stopped crashing this week.
a. $\quad \lambda w:\left|\left\{\mathbf{x}: \mathbf{c o m p u t e r}(\mathbf{x}, \mathbf{w}) \wedge \exists \mathbf{t}<\mathbf{t}_{\mathbf{0}} \operatorname{crashed}(\mathbf{x}, \mathbf{t}, \mathbf{w})\right\}\right| \geq \mathbf{1}$.
$\underline{\mid\left\{x: \text { computer }(x, w) \wedge \exists t<t_{0} \text { crashed }(x, t, w) \wedge \neg \operatorname{crashed}\left(x, t_{0}, w\right)\right\} \mid=1 .}$
b. $\approx$ The numbers of computers that went from crashing to not crashing is one

(15) Exactly one computer crashed again this week.

a. $\quad \lambda w:\left|\left\{\mathbf{x}: \operatorname{computer}(\mathbf{x}, \mathbf{w}) \wedge \exists \mathbf{t}<\mathbf{t}_{\mathbf{0}} \operatorname{crashed}(\mathbf{x}, \mathbf{t}, \mathbf{w})\right\}\right| \geq \mathbf{1}$.

b. $\approx$ The number of computers that just crashed is one

Both stop and again introduce an existential presupposition about crashing last week. Because stop entails its presupposition, evaluating the truth of (14) consists only in counting the number of Linux computers that didn't crash this week, since those are the ones that crashed last week to start with. Since only one of the Linux computers crashed this week, (14) is a true description of the situation. By contrast, again does not entail its presupposition and therefore considerations about last-week crashes do not factor in when evaluating the truth conditions of (15). Since more than one (namely two) of the four computers crashed this week (one in each group) (15) fails at giving an accurate description of the situation.

\subsection{Experimental support}

In a series of experiments testing sentences like (14) and (15) and controlling for various potential confounds, we found that English speakers' judgments align with Sudo's claims (Zehr 
and Schwarz 2016). ${ }^{6}$ We describe the design of these experiments below, as we used the same design for the experiment discussed in this paper. Results from Cummins et al. (2012) and Amaral and Cummins (2015) also provide indirect support for the view that the presuppositions of a subset of expressions make optional contributions at the truth conditional level. These researchers presented their participants with brief dialogues in which one interlocutor affirms a presuppositional question while denying the presupposition. The dialogues were reported to be more natural for some triggers than for others. Djärv et al. (2017) adapted their paradigm to investigate cognitive vs. emotive factives (16) and found that their participants rated the dialogues as more natural when they involved emotives. They offer an analysis where speakers can accept an utterance while denying its presuppositions as long as its truth conditions are met: such an attitude is blocked for cognitive factives, which the authors analyze as entailing their presuppositions, but it is available for emotive factives, which are analyzed as non-entailing triggers (17). ${ }^{7}$

$Q$ : Was Nadia aware cog $_{\text {/happy }}$ emo that the Patriots won the Superbowl?

$A$ : Yes, although they didn't.

$$
\begin{aligned}
& \text { Yes [truth conditions], although [not presuppositions]. } \\
& \lambda w: \operatorname{win}(\text { Patriots, } \mathbf{w}) . w \operatorname{win}(\text { Patriots }, w) \wedge \forall w^{\prime} \in \operatorname{Dox}(m, w) \text { win }\left(\text { Patriots }, w^{\prime}\right) . \quad \text { CoG } \\
& \lambda w: \operatorname{win}\left(\text { Patriots, w). happy }(m, w) \wedge \underline{\forall w^{\prime} \in \operatorname{Dox}(m, w) w i n\left(\text { Patriots }, w^{\prime}\right)} . \quad\right. \text { EMO }
\end{aligned}
$$

Experimental work on the processing of presuppositions provides us with further evidence that presuppositions come in different types. Domaneschi et al. (2014) invited their participants to memorize figures displayed on the screen for 6 seconds, listen to a recording of a short text, answer a question about the content of the text, and finally indicate which of sixteen figures displayed on the screen they were told to memorize at the beginning. The authors were interested in what happened between the two figure-display steps: the text contained five types of presupposition triggers, and five of the subsequent questions addressed their presuppositions. Having to keep in mind representations of figures for later recall constitutes a cognitive load that, the authors assumed, might unevenly impact the processing of presuppositions as opposed to the processing of entailments/truth conditions. Their participants' answers indicate that they consistently endorsed the presuppositions of definite descriptions and factive verbs: after listening to the text-which contained the zambezi sharks and the guide explained that all the sharks are female - the participants reported that there were zambezi sharks in the aquarium and that all the sharks were female. By contrast, their participants much less readily endorsed the presuppositions of focus-sensitive particles and iterative triggers: the text contained even the zambezi sharks are taken out of their tanks and the re-introduction of a male shark into the tank, but only slightly more than half the participants reported that other animals were sometimes taken out of the tank and that a male shark had previously been introduced into the tank. Tiemann (2014) and Tiemann et al. (2015) randomly presented each of their participants with

\footnotetext{
${ }^{6}$ Importantly, judgments in control conditions indicate that our design successfully blocked problematic widescope readings of again which would yield the following interpretation for (15): this week again, one computer crashed.

${ }^{7}$ We give over-simplified truth conditions for happy: the crucial point is that they do not entail the domain conditions/presupposition.
} 
one of two versions of a very short story: they both contained again and varied in whether they provided explicit support for its presupposition (18). An increase in reading times in the unsupported version indicates that participants were sensitive to the presence of a presupposition, and yet they show no sign of endorsement of the presupposition when probed for it (choosing to report that Linda received one pink lamp, not two).

Last week, Linda bought Judith a pink lamp for a room.

a. Two days ago, Judith received a pink lamp again.

SUPPORT

b. Two days ago, Linda received a pink lamp again.

NEUTRAL

Both groups of authors analyze their results in light of a proposal by Glanzberg (2005) and claim that presupposition triggers pattern in two categories, differing in the optionality vs. necessity to endorse the truth of their presuppositions for successfully processing the sentences in which they appear. This paper proposes to analyze the entailing vs. non-entailing distinction in the same terms. The next section describes Glanzberg (2005)'s proposal and formulates a hypothesis linking it to the entailment properties of presupposition triggers.

\section{The Removability/Independence Hypothesis}

Glanzberg (2005) argues for a model of utterance interpretation where only a subset of presuppositions have crippling consequences when not supported by context. He illustrates such a situation with cleft constructions (19), which are known to trigger an existence presupposition (19a). ${ }^{8}$ Glanzberg proposes that determining the non-presuppositional contribution of cleft constructions necessitates resolving their presuppositional contribution, insofar as the existence presupposition binds a variable that features in the truth conditions (19b). ${ }^{9}$ Being unable to instantiate the variable (because the context clashes with the existence presupposition) results in being unable to process the truth conditions. ${ }^{10}$

Was it Shappa who fixed the car?

a. Someone fixed the car

b. $\lambda w: \exists \mathbf{x} \operatorname{fixed}(\mathbf{C}, \mathbf{x}, \mathbf{w}) \cdot \underline{x=S}$.

On the other hand, Glanzberg proposes that determining the non-presuppositional contribution of too is independent from determining its presuppositional contribution, insofar as it involves no variable bound across the two domains (20b). As a result, even if the context establishes that the presupposition cannot hold (e.g., it has already been settled that Shappa did not fix anything-except possibly the phone) the truth conditions can still be felicitously processed.

\footnotetext{
${ }^{8}$ One might argue that clefts also carry exhaustivity presuppositions. We ignore this aspect here for the sake of the discussion.

${ }^{9}(19 b)$ exhibits non standard conventions for cross-domain anaphora: the $x$ appearing in the truth conditions is to be read as bound by the existential operator in the domain condition.

${ }^{10}$ Our use of the expression truth conditions diverges from Glanzberg's, as is our attempt at rendering the interpretation of presuppositional utterances in Heim and Kratzer (1998)'s notation style, since Glanzberg's analyses are termed in a dynamic semantic framework.
} 
Did Shappa fix the phone too?

a. Shappa fixed something else (e.g., the car)

b. $\quad \lambda w: \exists \mathbf{x} \neq \mathbf{P}$ fixed $(\mathbf{x}, \mathbf{S}, \mathbf{w})$. fixed $(P, S, w)$.

Glanzberg's approach provides a possible explanation for why a presupposition trigger would fall on one side or the other of Abusch's split, and the view that (part of) the presuppositional and non-presuppositional contents of some triggers would be inextricably intertwined resonates with the view that some triggers entail their presuppositions. At this point, it is a good idea to reiterate our goal: explain Abusch's observations about a contrast in presupposition suspension in terms of the Sudo/Klinedinst entailing vs. non-entailing distinction and explain the entailing vs. non-entailing distinction in terms of Glanzberg's intertwined vs. independent contributions distinction. However, we will depart from the methods Glanzberg uses to probe how each trigger relates its presuppositional and non-presuppositional contributions. ${ }^{11}$ Instead, we will formulate the Removability/Independence Hypothesis, which we think makes clear and intuitive predictions about which triggers entail their presuppositions and which do not.

\section{The Removability/Independence Hypothesis}

Presuppositions are NOT entailed if and only if removing triggering material yields (non-strictly) weaker interpretations of sentences

Let us use (22) to illustrate how this hypothesis predicts that the presupposition of again is not entailed. Removing again from (22a) results in (22b), which is a grammatical question whose interpretation is equivalent to (22a), minus the presupposition of the latter. According to the Removability/Independence Hypothesis, again does not entail its presupposition.

a. Did Aki's PC just crash again?

(i) Presupposed: Aki's PC crashed before

(ii) Questioned content: Aki's PC just crashed

b. Did Aki's PC just crash again?

(i) Presupposed: Aki's PC erashed before

(ii) Questioned content: Aki's PC just crashed

On the other hand, removing stop from (23a) results in (23b). Let alone the crucial syntactic role of stop as a matrix verb, no minimal syntactic reconstruction of (23b) (e.g., substituting is for did or removing -ing along with stop) would succeed in conveying the contrasting aspect of stop and in yielding an interpretation weaker or equivalent to that of the initial sentence.

a. Did Aki's PC stop crashing?

(i) Presupposed: Aki's PC crashed before

(ii) Questioned content: Aki's PC does not crash now

\footnotetext{
${ }^{11}$ Glanzberg starts from the observation that the flow of a discussion is differently impacted by unmet presuppositions from different triggers, but later refines his view on factive predicates. We will not pursue the same route, but note that results from Djärv et al. (2017), discussed earlier, hint at a contrast between emotive and cognitive factive predicates, which Glanzberg treats as equally intertwining their presuppositional and non-presuppositional contributions (after the necessary application of a complex repair strategy, see his Section V.4).
} 
b. *Did Aki's PC stop crashing?

(i) Presupposed: Aki's PC erashed before

(ii) Questioned content: ?? Aki's PC does not crash now

The two examples above should make clearer the intuition motivating the Removability/Independence Hypothesis: the semantic added value of some triggers, such as again, lies entirely in their presuppositions, so that their contribution is ultimately independent from the non-presuppositional content of the sentences in which they appear. Triggers like stop on the other hand, besides introducing presuppositions, make crucial contributions to the nonpresuppositional content of the sentences in which they appear, in such a way that one cannot identify a presuppositional component next to a non-presuppositional component: the two come as one block. The hypothesis states that triggers of the former type define truth conditions that are independent from their presuppositions, while the presuppositions of triggers of the latter type are entangled in their truth conditions.

It should be noted that the hypothesis refers to triggering material rather than directly to triggers. Such a formulation is particularly adapted to cases like emotive vs. cognitive factives, for which designating factive predicates as triggers is standard, even though some authors propose to locate the source of the presupposition in the complementizer material (Chierchia 2015). We do not commit to a particular position here, but our formulation has the welcome property of categorizing the emotive factive happy as non-entailing and the cognitive factive aware as entailing, as can be seen in examples (24) and (25).

a. Is Michelle happy that it's raining?

(i) Presupposed: it's raining

(ii) Questioned content: Michelle is happy (at the idea that it's raining)

b. Is Michelle happy that it's raining?

(i) Presupposed: it's raining

(ii) Questioned content: Michelle is happy (at the idea that it's raining)

(25) a. Is Michelle aware that it's raining?

(i) Presupposed: it's raining

(ii) Questioned content: Michelle believes that it's raining

b. ? Is Michelle aware that it's raining?

(i) Presupposed: it's raining

(ii) Questioned content: ?? Michelle believes that it's raining

In contrast to Glanzberg (2005)'s proposal, the Removability/Independence Hypothesis gives a prominent role to the form of triggering material. It is conceivable that two expressions make the same presuppositional and non-presuppositional contributions, but differ in that one but not the other has identifiable (and removable) material introducing presuppositions. As a result, the Removability/Independence Hypothesis expects that there should exist pairs of sentences whose truth conditions only differ in whether they entail their presuppositions. 


\section{Experiment}

We considered the three presuppositional expressions, go again, go back and return, which, paired with a destination, give rise to contextually equivalent effects: They all describe a visit while presupposing another previous visit. The two first expressions, however, differ from the last in a crucial way with respect to our hypothesis: they contain two clearly identifiable parts, one of which communicates about a visit ( $\mathrm{go}$ ) and one of which adds a presupposition of a previous visit (again/back). The contrast becomes evident when we apply the test introduced above: (26b) and (27b) define interpretations equivalent to (26a) and (27a) modulo the disappearance of their presuppositions; removing return from (28a) though results in the uninterpretable sentence ( $28 \mathrm{~b}$ ), where the main predicate is missing. ${ }^{12}$

(26) a. Did Dominique go to the shop again?

(i) Presupposed: Dominique previously went to the shop

(ii) Questioned content: Dominique went to the shop

b. Did Dominique go to the shop again?

(i) Presupposed: Dominique previously went to the shop

(ii) Questioned content: Dominique went to the shop

(27) a. Did Dominique go back to the shop?

(i) Presupposed: Dominique previously went to the shop

(ii) Questioned content: Dominique went to the shop

b. Did Dominique go baek to the shop?

(i) Presupposed: Dominique previously went to the shop

(ii) Questioned content: Dominique went to the shop

(28) a. Did Dominique return to the shop?

(i) Presupposed: Dominique previously went to the shop

(ii) Questioned content: Dominique went to the shop

b. *Did Dominique return to the shop?

(i) Presupposed: Dominique previously went to the shop

(ii) Questioned content: ?? Dominique went to the shop

Based on the Removability/Independence Hypothesis only return should entail its presuppositions. We tested this prediction by embedding the three triggers in an Exactly $N$ environment, as discussed in Sudo (2012). As mentioned in Section 2.3, we successfully designed experiments using Sudo's test in the past. The present experiment uses the same experimental design.

\footnotetext{
${ }^{12} \mathrm{~A}$ weaker version of the hypothesis analyzing return as non-entailing by identifying $r e$ - as the sole contributor of the presupposition would have to sacrifice transparency, for turn cannot be associated with the truth conditions of return, and it would ultimately fail at giving a criterion to identify material contributing only presuppositions.
} 


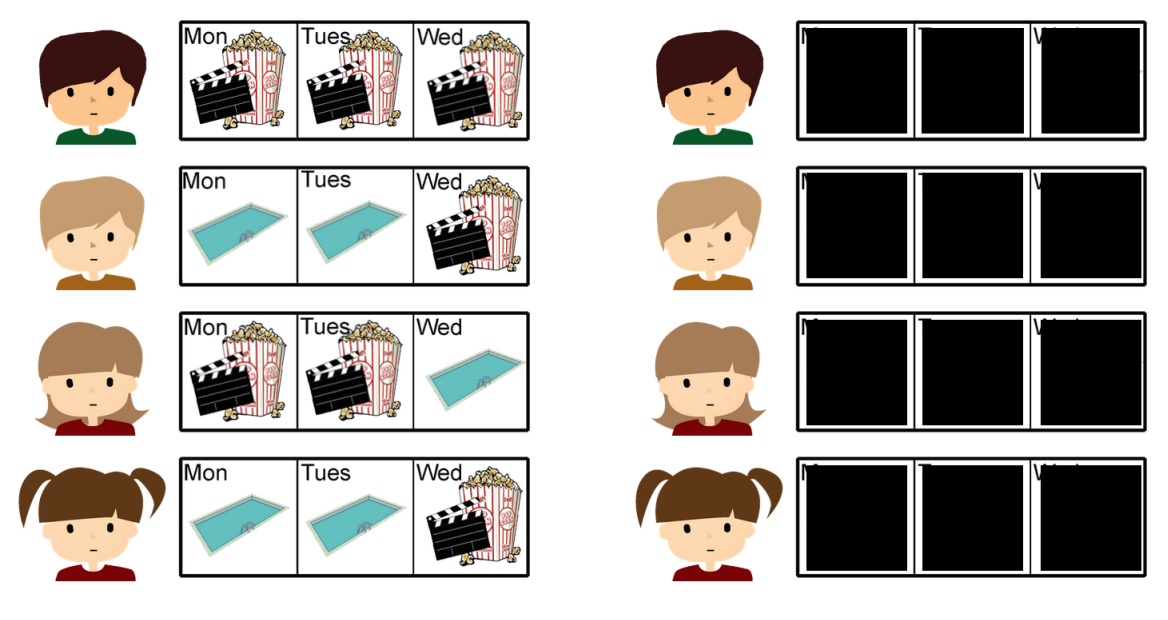

Figure 1: A visible and a covered picture displayed during a critical trial.

\subsection{Design}

Our experiment used the Exactly $N$ test described in section 2.2 in a Covered Box design. Participants were informed that their task was to associate a sentence with one of two pictures on the screen, one fully visible and one whose content was partially hidden. The pictures in Figure 1 illustrate the type of pictures on the screen during critical trials for the sentences in (29). If participants access an entailing representation of the sentence (i), then they should report the visible picture on the left as a good match, since only one of the four characters is presented as going to the movies both before and on Wednesday. On the other hand, the visible picture on the left does not match a non-entailing representation (ii) since three of the four characters went to the movies on Wednesday, and participants should therefore select the covered picture on the right on the reasoning that the activity schedules obstructed by the black squares must represent a better match for the sentence.

(29) a. Exactly one kid went to the Ore City movie theater again on Wednesday

b. Exactly one kid went back to the Ore City movie theater on Wednesday

c. Exactly one kid returned to the Ore City movie theater on Wednesday

(i) $\quad \mid\{x$ : movies $(x$, before $W) \wedge$ movies $(x, W)\} \mid=1$

(ii) $|\{x: \operatorname{movies}(x, W)\}|=1$

\subsection{Materials and Participants}

In addition to the triggers again, back and return, we included two other triggers to serve as baselines, stop and also (30), that we found in a series of previous studies to give rise to responses respectively consistent with an entailing representation and a non-entailing representation (Zehr and Schwarz 2016). We used Prolific.ac to recruit 150 participants, who we randomly assigned to one of the five triggers; they were paid $£ 1.5$ for an average duration of 12 minutes. Visual stimuli like that in Fig. 1 defined the Test condition: we code them as $A B A B \rightarrow A A B A$ to represent the transition from each character's Monday/Tuesday activities to their Wednesday activities ( $A$ standing for the activity mentioned in the Exactly one sentence). 
The code for the visual stimuli in the Visible Control condition was $A B A B \rightarrow A B B B ;{ }^{13}$ the code for the visual stimuli in the Covered Control condition was $A B A B \rightarrow A B A A .{ }^{14}$ Each participant saw 36 items, corresponding to 12 repetitions of each condition. We used a latin-square design to create 15 groups so that each of the 36 items would appear in all three conditions for all five triggers but each participant would see each item for only one condition. The items were presented in random order.

a. Exactly one kid stopped going to the Ore City movie theater on Wednesday
b. Exactly one kid went to the Ore City movie theater again on Wednesday

Each trial consisted of a sequence corresponding to the following script. The two visible (left) and covered (right) pictures appear centered on the screen with only the Monday and Tuesday activities visible. An audio recording of a context sentence like that in (31) automatically plays back while participants look at the screen. The Wednesday slots appears at the end of the playback (covered by a black squares for the picture on the right) and an audio recording of one test sentence like the ones in (30) automatically starts playing back. From this point on, participants can select the visible left picture by pressing the $F$ key on their keyboard, or the covered right picture by pressing $J$. The screen is cleared and the next trial starts after one of these two keys is pressed.

(31) This week, these kids went to Ore City for the first time. At the beginning of the week, some kids went to the Ore City pool, and some people went to the Ore City movie theater.

The sentences were recorded by a native speaker of English who was instructed to produce the intonation contour on the also sentences in such a way that it conveyed an association with on Wednesday. ${ }^{15}$

\subsection{Predictions}

Also and stop served as baselines. We reported in Zehr and Schwarz (2016) that also sentences yielded covered image choices and stop sentences visible image choices in the Test condition, as consistent with the predictions made by a view where stop, but not also, entails its presuppositions. We saw at the beginning of this section how the Removability/Independence Hypothesis analyzes return as an entailing trigger and back and again as non-entailing triggers. It thus predicts that return patterns with stop in eliciting visible picture choices in the Test condition, and that back and again pattern with also in eliciting covered picture choices.

\footnotetext{
${ }^{13}$ Note that in the Visible Control condition two of the four characters engage in the mentioned activity on Monday and Tuesday, thus controlling for potential wide-scope readings of also and again (see footnote 6).

${ }^{14}$ The codes defining the condition for stop were: $A B B A \rightarrow B B B A$ (Test), ABBA $\rightarrow B A A A$ (Visible Control) and $A B B A \rightarrow B A B B$ (Covered Control)

${ }^{15} \mathrm{An}$ archived version of the experiment can be found at http://spellout.net/ibexexps/ SchwarzLabArchive/PsEntStopReturnBackAgain/experiment.html.
} 


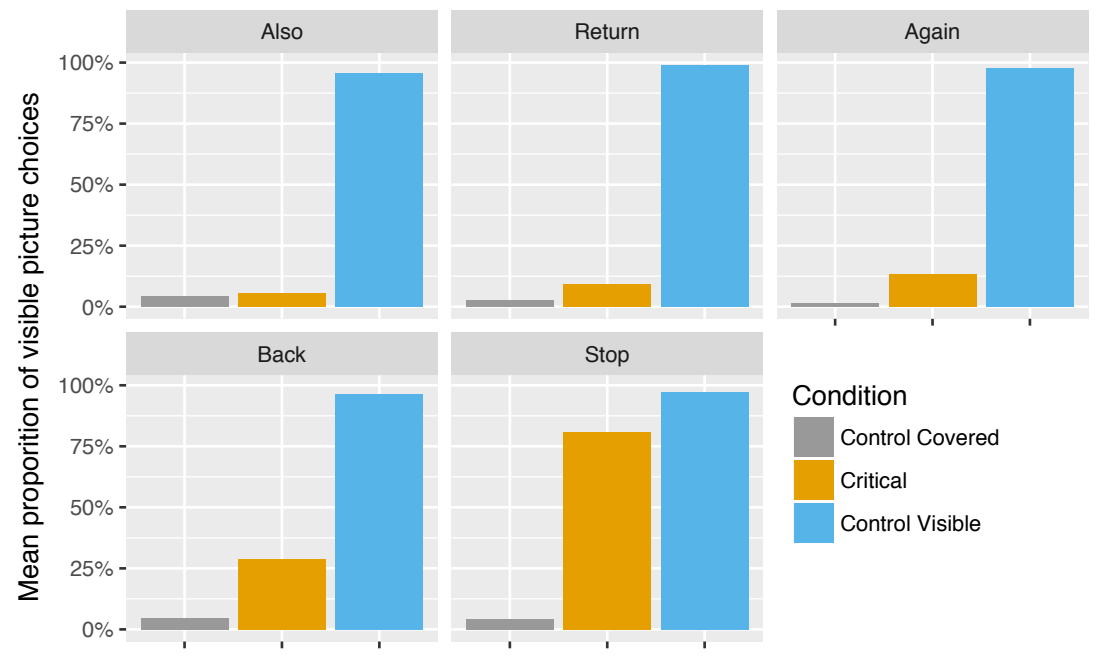

Figure 2: Proportions of visible picture choices per trigger per condition. Visible choices in critical conditions (green) are analyzed as indication of contribution to the entailments.

\subsection{Results}

Accuracy on controls was good overall, with the exception of a few participants in each group. We excluded from our analyses any participant who chose more than $25 \%$ visible or covered pictures in the Control Covered or Control Visible conditions respectively, ${ }^{16}$ for a final set of 128 accurate participants out of 150. The bar plot in Figure 2 reports the mean choice of visible pictures for each trigger in each condition for the accurate participants.

We used the $R$ software (version 3.3.3) and the function lmer (version 1.1 - 13) to fit a logistic regression model on the data, predicting choices of visible pictures as a function of two factors (baselines first): Condition (Test vs. Control Covered vs. Control Visible) and Trigger (Also vs. Return vs. Again vs. Back vs. Stop). The model tested both for simple effects and interactions between the two factors and included a random intercept per item and per participant as well as a random slope per participant per condition. Following the recommendations of Bates et al. (2015) we started from a maximal random structure and simplified it until we reached the simplest converging model that would not significantly differ in goodness of fit (as reported by ANOVA comparisons of models). Our final model forced a zero correlation in the random slope per participant per condition.

The model reports no significant contrast between Also-Test on the one hand and Return-Test $(\beta=1.0181 ; S E=1.285 ; p=0.428)$ and Again-Test $(\beta=1.8218 ; S E=1.338 ; p=0.173)$ on the other hand. Back-Test, however, significantly increased the likelihood of a visible picture observation $(\beta=3.539 ; S E=1.349 ; p<0.01)$ and so did Stop-Test $(\beta=11.2195 ; S E=$ $1.849 ; p<0.01)$. Participants were reportedly more likely to choose the visible picture in AlsoControl Covered than in Also-Test $(\beta=2.9478 ; S E=1.158 ; p<0.05)$. Note that this effect is opposite of the descriptive summary in Fig. 2. Looking at individual profiles reveals that 9

\footnotetext{
${ }^{16}$ Distributed across trigger groups as follows: Also: -7 ppts; Return: -1 ; Back: -3 ; Again: -4; Stop: -7 .
} 
out of the 24 Also participants selected the visible picture at least once (and only once for 7 of them) in the Control Covered condition contra only 6 in the Test condition (4 of them were also Control Covered acceptants). The descriptive flip in the bar plot seems to be entirely due to one participant who selected the visible picture on more than half the Test trials, whereas no participant did so on more than a quarter the Control Covered trials (which follows from our filtering for accuracy). Two significant interactions are noteworthy: Also vs. Again $\times$ Test vs. Control Covered $(\beta=-2.8881 ; S E=1.4239 ; p<0.05)$ and Also vs. Back $\times$ Test vs. Control Covered $(\beta=-3.4490 ; S E=1.3824 ; p<0.05)$. Looking at individual profiles is again informative: 2 out of the 26 Again participants always chose the visible picture in the Test condition (while no Also participant did) and 9 out of the 27 Back participants chose the visible picture more than half the time in the Test condition (only one Also participant did).

\section{Discussion}

The results clearly contradict the predictions of the Removability/Independence Hypothesis: return did not pattern with the entailing trigger stop. Instead, it behaved remarkably similarly to the non-entailing trigger also, as did again and back. At the same time, it is important to note that these results replicate the contrast between stop and also that we had found in previous studies, which can be explained by a contrast in entailment. The also participants rejected visible pictures that depicted more than one character engaging in the mentioned activity on Wednesday (Test and Control Covered conditions) regardless of their activities on Monday and Tuesday. The entailed presupposition of stop, however, factored into the truth conditions of the exactly one sentences, so participants considered the Monday and Tuesday activities when counting characters. As a result, they accepted visible pictures that depicted more than one character not engaging in the mentioned activity on Wednesday, as long as only one of them had previously engaged in it on Monday and Tuesday.

Though the Stop participants clearly contrasted with the others in overwhelmingly choosing the visible picture in the Test condition, a small subset of the latter also consistently chose the visible picture in the Test condition. This is not straightforwardly expected if also, again, back and return do not entail their presuppositions, as suggested by the majority of participants who rejected the covered picture. In section 2.1 we followed Klinedinst (2016) in introducing the operation of presupposition deletion as a one-step alternative to the two-step operation of presupposition conversion commonly known as local accommodation. The two operations are not mutually exclusive, and we analyze (rare) acceptance of the visible picture in the Test condition as resulting from local accommodation of the presuppositions of also, again, back and return. As a result of this operation, these triggers yield interpretations where the presupposition becomes part of the truth conditions, as is the case lexically for stop, and those participants who applied local accommodation accordingly chose the visible picture in the Test condition, where only one character engaged in the mentioned activity both on Wednesday and on Monday and Tuesday. The question remains as to what led some of our participants to access local accommodation interpretations of our sentences, and more particularly why such interpretations were more readily available for back (and, to a lesser extent, for again) than for also and return. We have to leave this question for future investigations. ${ }^{17}$

\footnotetext{
${ }^{17}$ Focusing presupposition triggers could favor local accommodation readings, and one could tentatively explain
} 
The Removability/Independence Hypothesis offered an explanatory approach by predicting which triggers entail and which do not entail their presuppositions. That return patterned with also rather than stop strikingly invalidates the hypothesis and leaves us in need of an account for why, out of the five triggers we tested, only stop seemed to always factor its presupposition into the process of picture selection. Tonhauser et al. (2013) conducted a series of three experiments showing that prosody influences whether factive presuppositions project outside of entailment-canceling environments, and offered an analysis where prosody serves as a proxy for the contextual information structure, which ultimately determines whether a presupposition is even triggered to start with. Following an analysis of our results along these lines, one would expect our stop recordings to manifest specific prosodic cues that distinguish them from our other recordings. We conducted post-hoc analyses focusing on pitch. Our hypothesis was that a high pitch on a trigger draws attention to its contributions so that its presuppositions end up part of the truth conditions of the sentence. The resulting prediction was that higher pitches should increase the likelihood of a visible picture choice (because what happened on Monday and Tuesday becomes more salient). We measured the mean relative pitches on each trigger in our recordings and found a higher average pitch for back than for again, as consistent with the observation that back yielded more visible picture choices than again. The mean pitch for stop was even higher, but so was the mean pitch for return (and to a lesser extent the mean pitch of also) which, however, yielded fewer visible picture than both back and again. Therefore, pitch alone clearly cannot explain our observations, but it could account for some variation in our data (see footnote 17), in line with Djärv and Bacovcin (2017), who conducted an experiment as a response to Tonhauser et al. (2013) and argued that prosody is a real but a small factor influencing the status of presuppositions.

Abrusán (2016) offers an account of presuppositions where temporal reference plays a central role. She proposes a typology where triggers can differ in whether they refer to a single or to multiple reference times. The presuppositions of triggers referring to a single reference time have to be considered jointly with non-presuppositional content because they necessarily refer to the same reference time; other triggers introduce a second reference time in their presuppositions and thus make two distinct, independent contributions. It seems that stop refers to a unique time span: stopping consists in reaching an end point on a temporal scale. By contrast, return, (go) back, (go) again and also (go on Wednesday) all refer to a distinct past reference time, and impose no continuity relation with their main reference time. As a result, it would be impossible to ignore the presupposition of stop when making a decision about how many characters satisfy the description, whereas it would be possible to focus on the salient event of going in the other cases. Data from a pilot and from a new experiment, however, suggest that continue, which is a prototypical case of a continuous (and thus single) reference time as in the case of stop, patterns along with non-entailing triggers in the exactly one test.

Closely related to the idea of (dis)continuity is an explanation in terms of contrastivity. As alert readers may already have noticed, stop stands out in being the only trigger that involves a mismatch between the Monday and Tuesday activities and the Wednesday activity. A natural

the higher frequency of local accommodation for again and back under the assumption that moving them to focus position was easier than moving also (which came with a particular association contour in our recordings) and return (which is a main predicate). See below for further discussion on the role of prosody in our experiment. 
explanation for our results would be that this property of stop led the participants in the corresponding group to pay attention to the Monday and Tuesday slots throughout the task, while the participants in the other trigger groups could have decided to exclusively rely on the Wednesday slots to make their decisions. ${ }^{18}$ Such a task-and-trigger-specific strategy is highly problematic, since it would undermine our ability to diagnose the entailing properties of a trigger through the exactly one design. Initial results coming from a follow-up experiment including a condition where the Monday and Tuesday slots in the visible picture do not even satisfy an existential presupposition reveal that many participants indeed do not pay attention to it (i.e., they choose the visible picture despite no character satisfying the presupposition). But they also reveal clearly different profiles for stop and again participants: while no participant who paid attention to Monday and Tuesday ever chose the covered picture for Stop-Test, some did for Again-Test. This suggests that even when Again participants are actively looking at Monday and Tuesday (as reflected by rejection of pictures not satisfying the existential presupposition) some of them still are unhappy with pictures in which more than one character engages in the mentioned activity on Wednesday, even though only one also engaged in it on Monday and Tuesday. Moreover, the follow-up experiment also included the triggers no longer and not anymore which share the contrastivity of stop, and yet they did not show a pattern specific of entailing triggers. Our current project is to investigate the possibility that contrastivity might interact with continuity in impacting the truth conditional contributions of presuppositions.

\section{Conclusion}

By formulating the Removability/Independence Hypothesis, we explored a possible explanatory account of the hard vs. soft split. In doing so, we combined the Sudo/Klinedinst entailing vs. non-entailing account of the contrast in local contribution of soft vs. hard presuppositions, with an approach inspired by Glanzberg, whereby the fulfillment of some presuppositions is a necessary condition to arrive at interpretable utterances. In contrast to competing explanatory approaches, this hypothesis has the particularity of giving a formal, rather than conceptual, identification criterion for hard vs. soft triggers. Consistently, it sorts the presuppositional expressions return and go back into opposite sides of the split, even though they express nearly equivalent concepts. The results of our experiment allow us to rule out this formal hypothesis, and thus indirectly provide support for conceptual approaches. ${ }^{19}$ The question of what determines the typological properties of a presupposition trigger has important repercussions. Perhaps the most important one concerns language acquisition: if each presupposition trigger belongs either to the hard or to the soft category, how do children eventually figure out which box a specific expression should go into? The question appears even more intricate in light of Dudley's (2017) observation that cues that an expression is presuppositional are very scarce in children corpora. Identifying a conceptual source for the split would help shed light on how children arrive at (or maybe start with) a mature representation of presupposition triggers.

\footnotetext{
${ }^{18}$ Note that, by design, picture selection would be unaffected whether the Monday and Tuesday slots were ignored in the control conditions.

${ }^{19}$ Though formal considerations might still be in order when accounting for the higher rate of readings of back in which the presupposition makes a local contribution.
} 


\section{References}

Abrusán, M. (2016). Presupposition cancellation: Explaining the 'soft-hard' trigger distinction. Natural Language Semantics 24(2), 165-202.

Abusch, D. (2002). Lexical alternatives as a source of pragmatic presuppositions. In B. Jackson (Ed.), Semantics and Linguistic Theory (SALT) 12, Ithaca, NY, pp. 1-19. Cornell University.

Amaral, P. and C. Cummins (2015). A Cross-Linguistic Study on Information Backgrounding and Presupposition Projection, pp. 157-172. Cham: Springer International Publishing.

Bates, D., R. Kliegl, S. Vasishth, and H. Baayen (2015). Parsimonious mixed models. arXiv preprint arXiv:1506.04967.

Chierchia, G. (2015). Factivity meets polarity. Berkeley Linguistics Colloquium.

Cummins, C., P. Amaral, and N. Katsos (2012). Experimental investigations of the typology of presupposition triggers. Humana Mente 23, 1-15.

Djärv, K. and H. A. Bacovcin (2017). Prosodic effects on factive presupposition projection. In Proceedings of SALT 27, pp. 116-133.

Djärv, K., J. Zehr, and F. Schwarz (2017). Cognitive vs. emotive factives: An experimental differentiation. In Proceedings of Sinn und Bedeutung, Volume 21. Semantics Archive.

Domaneschi, F., C. Penco, E. Carrea, and A. Greco (2014). The cognitive load of presupposition triggers: Mandatory and optional repairs in presupposition failure. Language, Cognition and Neuroscience 29(1), 136-146.

Dudley, R. (2017). The role of input in discovering presupposition triggers: Figuring out what everybody already knew. Ph. D. thesis, University of Maryland.

Gajewski, J. R. (2011). Licensing strong npis. Natural Language Semantics 19(2), 109-148.

Glanzberg, M. (2005). Presuppositions, truth values and expressing propositions. In G. Preyer and G. Peter (Eds.), Contextualism in philosophy: Knowledge, meaning, and truth, pp. 349396. Oxford, UK: Oxford University Press.

Heim, I. (1983). On the projection problem for presuppositions. In M. Barlow, D. Flickinger, and N. Wiegand (Eds.), Proceedings of WCCFL 2, pp. 114-125. Stanford University.

Heim, I. and A. Kratzer (1998). Semantics in Generative Grammar. Blackwell.

Klinedinst, N. (2016). Two Types of Semantic Presuppositions, pp. 601-624. Cham: Springer International Publishing.

Sudo, Y. (2012). On the Semantics of Phi Features on Pronouns. PhD thesis, Massachusetts Institute of Technology, Cambridge, MA.

Tiemann, S. (2014). The processing of 'wieder' ('again') and other presupposition triggers. $\mathrm{Ph}$. D. thesis, Universität Tübingen.

Tiemann, S., M. Kirsten, S. Beck, I. Hertrich, and B. Rolke (2015). Presupposition Processing and Accommodation: An Experiment on wieder ('again') and Consequences for Other Triggers, pp. 39-65. Cham: Springer International Publishing.

Tonhauser, J., D. Beaver, C. Roberts, and M. Simons (2013). Towards a taxonomy of projective content. Language 89(1), 66-109.

Zeevat, H. (1992). Presupposition and accommodation in update semantics. Journal of Semantics 9(4), 379-412.

Zehr, J. and F. Schwarz (2016). Entailed vs. non-entailed presuppositions - an experimental assessment. In Proceedings of NELS 46, Amherst, MA. GLSA Publications. 